\title{
Systematic Review of Computational Models for Human Brain Parcellation
}

\section{Patrick Owate ${ }^{1,2}$, Benjamin Aribisala', Charles Uwadia² and Philip Adewole ${ }^{2}$}

${ }^{1}$ Department of Computer Science, Faculty of Science, Lagos State University, Nigeria

${ }^{2}$ Department of Computer Sciences, Faculty of Science, University of Lagos, Nigeria

\begin{abstract}
:
Introduction: The human brain consists of four main lobar regions: Frontal lobe, Parietal lobe, Temporal lobe and Occipital lobe. Most of the existing models used for the parcellation of brain into these lobes have limited accuracy when applied to ageing brain.

Aims: To systematically review the existing models of parcellating brain Magnetic Resonance Images, their strengths and weaknesses, and the possibility of using them for ageing brain.

Materials and Methods: PubMed was searched combining search terms for Parcellation, Brain and Magnetic Resonance Imaging (MRI). Articles were considered if they met the following criteria: Parcellation method was indicated, imaging technique was MRI, high resolution anatomical T1Weighted was used, lobar regions were parcellated, number of lobar regions was indicated.

Results: The search resulted into 569 articles. 174 articles ( 7 from the list of references) were potentially relevant and their abstracts were read. Out of these, 108 were not relevant because they either focused on animal studies, sub-cortical segmentation or tissue segmentation. The full papers of the remaining 66 were reviewed. 39 articles met the inclusion criteria. Various parcellation models were reviewed and summarized into six groups: supervised learning, unsupervised learning, region growing, shape and appearance, energy-based and atlas-based models.

Conclusion: All the existing models identified were developed for parcellation of young adult brains and none of them used age-related information. Atlas-based model was found to perform the best among all the models. Future work should consider extending atlas-based model by including ageing information which could make them perform well on ageing brain.
\end{abstract}

To Keywords: Parcellation, Human Brain, Lobar regions, Models, MRI. 


\section{INTRODUCTION}

Human brain contains different regions with each region performing specific functions. In neuroimaging, studying a particular region of the brain requires identification of that region. Human brain consists of four main and distinct lobar regions, viz. Frontal lobe, Parietal lobe, Temporal lobe and Occipital lobe. Regional identification of the lobar regions and analysis of brain Magnetic Resonance Images (MRI) usually requires parcellation or segmentation of the brain into these lobar regions. This is useful for the diagnosis and evaluation of the neurological diseases such as stroke [1].

There are two major methods for parcellating the brain, viz. manual and automated methods. Manual parcellation, when done by an expert, is done by manually tracing the hypothesized region. Manual parcellation is accepted as more accurate parcellation method. In view of this, it is used for generating the ground truth which is used for assessing the accuracy of other parcellation techniques [2]. However, manual methods are time consuming (it can take a whole day for a trained neuro-radiology to parcellate one brain). They also have the problems of repeatability, subjectivity, and intensive human resource requirement. In view of these, automated method is preferred[3]. With automated method, computer algorithms can be used for parcellation of many brain images within a short period of time.

Identification of an automatic parcellation model that will combine accuracy, speed and objectiveness is a top research priority in neuroimaging. Various computer models have been proposed for parcellation of human brain into lobar sections but most of the existing models were developed for young adults and hence have limited accuracy during the parcellation of ageing brains. Parcellation of ageing brains presents a significant challenge to any computerized model because age-related changes such as brain atrophy [4], skull thickening, presence of white matter lesions and infarcts [5] increase inter-individual variability [2]. The focus of this study is hence, to assess the weaknesses and strengths of existing models, and to identify a robust computerized parcellation model that could be extended to allow parcellation of an ageing brain accurately, objectively and in a timely manner with minimum human intervention.

\section{MATERIALS AND METHODS}

PubMed was searched using search terms related to Parcellation, Brain and MRI. The search was conducted in English and only the papers that met the search criteria were reviewed. The original search term included Parcellation, Brain and MRI. Given that the focus of the study was on parcellation of anatomical brain MRI images, exclusion criteria were formulated to exclude articles that focused on brain connectivity and
Positron Emission Tomography (PET) and Diffusion Tensor Imaging. That lead to the modification of the search term to yield the final search term which was [(parcellation brain mri) NOT connectivity NOT functional NOT diffusion NOT PET]. Using this search term, some irrelevant articles were removed. Finally, more articles were obtained from the list of references of the articles retrieved using the final search term.

The full-text of all the potentially relevant papers were retrieved and reviewed. An article was considered relevant if it satisfies the following inclusion criteria.

(1) Parcellation model must be clearly indicated or implied.

(2) The use of high resolution anatomical T1-Weighted must be indicated or implied.

(3) The parcellation must be for the cortical regions (precisely, lobar regions) and not subcortical regions.

(4) The number of regions a brain is divided into must be indicated or implied.

The following were recorded for all the relevant articles: Authors name, Title, Date published, Journal name, Parcellation model, Boundary definitions, Age range, Sample size, Study population, Imaging technique, Type of Image, Orientation used, Aim of project and Contribution to knowledge.

Next, the articles were grouped according to the parcellation models used and the performance of each group of models was noted. Each group was carefully analyzed to identify the possibility of extension to handle ageing brain.

\section{RESULTS AND DISCUSSION}

\subsection{Results from Literature Search}

We used the Preferred Reporting Items for Systematic Reviews and Meta-Analyses (PRISMA, Figure 1) for the review [6]. The basic search resulted into 569 papers. Application of the exclusion criteria to remove papers that focused on connectivity, functionality, diffusion and PET reduced the papers to 167 . Through references or paper cited in the 167 papers additional 7 papers were identified, giving a total of 174 potentially relevant papers. After screening the abstracts of the 174 potentially relevant papers, 66 papers satisfied the criteria for the retrieval of their full-text version and these were retrieved. Out of these, 39 papers met inclusion criteria. 


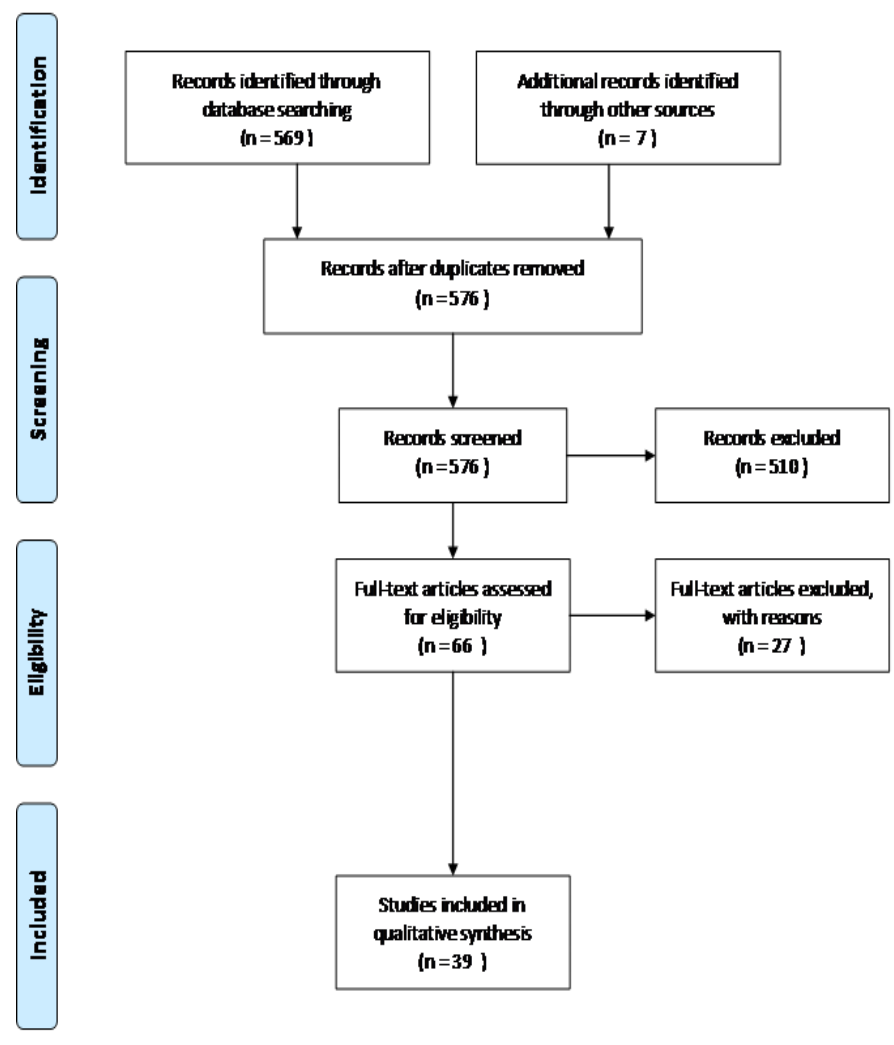

Figure 1: Preferred Reporting Items for Systematic Reviews and Meta-Analyses (PRISMA) flow diagram of included studies.

\subsection{Categorization of Automated Models for Brain Parcellation}

The models identified were categorized into six groups based on the algorithms used, namely Atlas-based [710], Supervised Learning models [1], Shape and Appearance models [11, 12], Energy-based models $[13,14]$, Unsupervised Learning models [15] and Region Growing models [15]. Grouping was done using the following rules: (1) An article is grouped under Atlasbased model if parcellation is based on the inference from manually labeled MRI brain image (called atlas in Neuroimaging) or training data by clinical experts. Also, atlas based method requires the use of registration algorithm. (2) An article was classified as using Supervised Learning model if parcellation employed machine learning technique, that is based on developing algorithm from training data and subsequently using the algorithm to parcellate the brain MRI. (3) An article is grouped under Shape and Appearance model if it uses shape relation and individual transformations to carry out parcellation on each structure of brain MRI. (4) An article belongs to Energy-based model if it carries out parcellation based on similarity (homogeneity) of image regions. (5) An article is grouped under Unsupervised Learning model if it does not make use of training data to parcellate, but uses model driven or statistical approach that does not require humans to set parameters.(6) An article is grouped under Region Growing model if it uses seed and homogeneity criteria to carry out parcellation.

\subsubsection{Supervised Learning Models}

This group of models uses machine learning technique called supervised learning to parcellate brain MRI by developing algorithm from training data and subsequently using the algorithm to parcellate the brain MRI. In supervised learning, the user will be required to set some parameters or supervise the parcellation to ensure acceptable degree of accuracy. An example of this group of models is parcellation of brain using Geometric Moment Invariants and Artificial Neural Network [1]. The strength of this model are: (1) It is not sensitive to the choice of the training data. (2) It works fine in noisy images. The major weakness of this model is that it cannot cope with the contrasts that exists in the slices of MRI [15]. Also, the method depends on parameter setting, so if the parameters are not set properly, the results may not be good.

K-Nearest Neighbour (KNN) [16] classifier is also a member of the Supervised Learning Models. It carries out parcellation by classifying voxels into different classes using the features of the voxels that are peculiar to that class. The strength of this method is that it is of higher accuracy and productivity than the parametric method, while the weaknesses are: (1) Its implementation is slow. (2) Its computational cost is very high. (3) The accuracy of this model can be decreased by the existence of noise or irrelevant features $[15,17]$.

Another model that belongs to this group is Bayesian Classifier that carries out parcellation using Bayes' theorem that the probability of an event is based on prior knowledge of conditions that might be related to the event $[15,16]$. The strength of this model is that it is fast and accurate if initialization is correct and the weaknesses are: (1) There is no robust method for initialization. (2) Convergence is usually problematic. (3) It is computationally expensive and intensive.

\subsubsection{Unsupervised Learning Models}

This group of models makes use of unsupervised machine learning technique to parcellate brain MRI. Unlike the supervised learning models, these models do not make use of training data. Since no training data is deployed, it becomes pertinent to design algorithms that interleave classification and estimation of the class or specific properties for this model. Examples of algorithms that belong to this model are fuzzy $\mathrm{C}$-means (FCM), K-means and thresholding methods [15].

The strength of thresholding method is that it is very effective and simple for images with various intensities while the weakness is that threshold value cannot be defined for multichannel images $[15,18]$. The strength of $\mathrm{K}$-means method is that it is fully automated while the 
weaknesses are: (1) It is computationally difficult. (2) The wrong choice of $\mathrm{k}$ (i.e. the number of clusters) can lead to inaccurate results $[15,19]$. The strength of Fuzzy $\mathrm{C}$-means is that it minimizes the objective function which results into the optimization of resources deployed for parcellation and the weakness is that it is significantly inefficient if it is used for noisy images and artifacts $[15,20]$.

\subsubsection{Region Growing Models}

In region growing, parcellation commences with a seed. The size of this region will be augmented by adding the pixels in the neighbour of the original seed based on homogeneity criteria [21]. The strength of these models is that their parcellation results are more accurate than those of pixel-based models and the weaknesses are: (1) They are not fully automatic techniques, and they need user interaction to select a seed. (2) The result of the parcellation process is usually affected by the choice of the seed points. (3) These methods also fail in obtaining acceptable results in natural images. (4) These methods only work well in homogeneous regions. (5) The extracted regions might contain some holes or discontinuities because these methods are sensitive to noise. (6) The partial volume effect (PVE) may cause different regions to become connected, which might lead to very wrong results.

\subsubsection{Shape and Appearance Models}

These models use shape relation and individual transformations to carry out parcellation on each structure of brain MRI. An example of this group of models is Constrained Optimization of Nonparametric Entropy-Based Segmentation. The strength of this method is that it does not depend on parameters and is robust [22] and the weakness is that image quality e.g contrast must be very good before accuracy can be assured. Another example of shape and appearance model is Mutual Information in Coupled Multi-Shape model. This is an extension of Shape-Based Deformable Active Contour Model. This model has an additional feature of being able to segment multiple shapes simultaneously in a seamless way [23]. The strengths of this method are: (1) It captures important co-variations among the different shape classes. (2) Displays a wide range of shape variability. (3) Handles large amount of additive noise. The weakness of this method is that image quality e.g contrast must be very good before accuracy can be assured.

\subsubsection{Energy-based Models}

These models use similarity (homogeneity) of image regions. The strength of the models is that they do not depend on parameters, i.e. they are nonparametric. The weakness of the models is that they can only take care of structures that have different intensity distribution from most of the surrounding tissues [24].

\subsubsection{Atlas-based Models}

Atlas-based models for parcellating brain MRI is based on the inference from manually labeled training images by Neuroimaging expert (Figure 2). The first stage of this method is the construction of the atlases. After this, the atlas can then be used to parcellate the target image. Parcellation can be done at atlas or subject's native space [2]. Atlas based parcellation requires the use of registration algorithm to normalize the differences in size and shape between the atlas and the individual's brain. Registration uses linear or non-linear with non-linear rated as better than linear [7]. Another important step in atlas based method is the choice of atlas. Single or multiple atlas could be used with the latter being more accurate than the former [7, 10, 25].

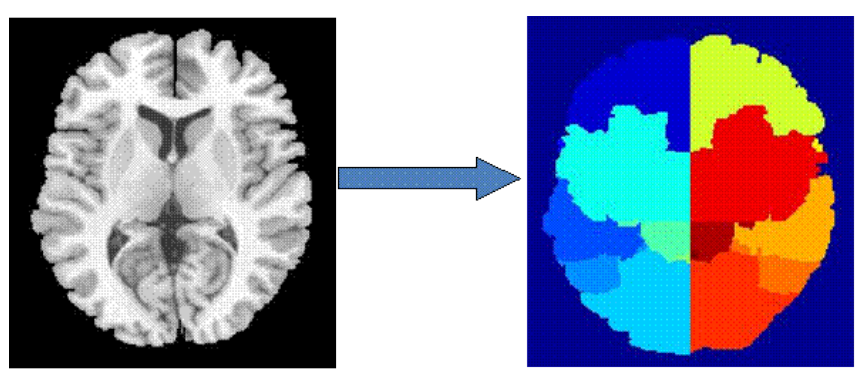

Figure 2: Images showing a T1W brain MRI image manually parcellated into pairs of right and left inferior frontal lobe, superior frontal lobe, temporal lobe, temporal-occipital lobe, occipital lobe, temporal-parietal lobe, parietal lobe and the cerebellum.

Source: Aribisala B.S., 2009, ISMRM Honolulu, U.S.A. [3].

When multi-atlas based parcellation model is deployed, the choice of atlases, the number of atlases and the method used for combining the atlases influence the final outcome of the experiment $[7,10,26]$. A very reliable method for selecting atlases is image similarity metrics (e.g. normalized mutual information or correlation ratio) $[7,10]$. In order to obtain the optimum number and combination of atlases, the use of classifier fusion based on majority vote has been suggested and shown to be an accurate method [7]. However, even when we use these reliable methods to select atlases along with the very best registration method, the effects of age-related atrophy could still create problems for automated parcellation methods.

\subsubsection{Strengths of Atlas-based Models}

1. One of the strengths atlas-based models have over other models of parcellation is the fact that atlas-based models can parcellate images with no well defined relation between regions and pixels' intensities. This is useful when objects of the same texture need to be 
parcellated or when there is lack of border or excessive noise.

2. The atlas-based parcellation will work well if the information about the differences between the objects is in spatial relationship between them or in other objects.

3. Other strength of atlas-based models is their use in clinical practice. These methods are used to measure the shape of an object or detect morphological differences between patients groups in computer aided diagnosis [27].

\subsubsection{The weaknesses of Atlas-based Models}

1. The time required for atlas construction is very enormous.

2. Since the atlas constructions are done by humans, there may be some level of subjectivity in the output of such atlases.

3. The performance of these models depends on the method of registration deployed.

4. Atlas selection and combination methods could also affect the performance of the models.

\section{CONCLUSION}

We systematically reviewed many relevant computational models for parcellating brain MR images. The methods were classified into six groups based on the types of algorithms used. The six groups are supervised learning models, unsupervised learning models, region growing models, shape and appearance models, energy-based models, and atlas-based models. It was established that among all these models, atlas-based models perform the best. However, atlas based method's performance could be improved if it uses some ageing related information. Future work should consider extending atlas based method by including ageing related information.

\section{ACKNOWLEDGEMENTS}

The authors would love to thank Mr. Mayowa and Mr. Bamiro of Computer Science Department, Lagos State University for their help during laboratory experiments, most especially the installation of the tools used for the experiment. We also appreciate Mr. Mohammed, Mrs. Omobolaji and Mr. Odun of the same department for all the help in the area of administrative tasks.

\section{AUTHORS' CONTRIBUTIONS}

All authors read and approved the final manuscript.

\section{REFERENCES}

[1] Moghaddam MJ, Soltanian-Zadeh H. Automatic segmentation of brain structures using geometric moment invariants and artificial neural networks.
Information processing in medical imaging : proceedings of the conference 2009;21:326-37.

[2] Aribisala BS, He J, Blamire AM. Comparative Study of Standard Space and Real Space Analysis of Quantitative MR Brain Data. Journal of Magnetic Resonance Imaging 2011;33:1503-9.

[3] Aribisala BS, Blamire AM. Comparison of Analysis of Brain Relaxation Times in Standard Space with Analysis in Individuals' Real Space. Proc. International Society for Magnetic Resonance in Medicine (ISMRM) 17, Honolulu, USA; May 2009. p. 4486

[4] Appelman APA, Exalto LG, van der Graaf Y, Biessels GJ, Mali W, Geerlings MI. White Matter Lesions and Brain Atrophy: More than Shared Risk Factors? A Systematic Review. Cerebrovascular Diseases 2009;28:227-42.

[5] Appelman APA, Vincken KL, van der Graaf Y, Vlek ALM, Witkamp TD, Mali WPTM, et al. White Matter Lesions and Lacunar Infarcts Are Independently and Differently Associated with Brain Atrophy: The SMART-MR Study. Cerebrovascular Diseases 2010;29:28-35.

[6] Moher D, Liberati A, Tetzlaff J, Altman DG, Group $P$. Preferred reporting items for systematic reviews and meta-analyses: the PRISMA statement. PLoS medicine 2009;6:e1000097.

[7] Aljabar P, Heckemann RA, Hammers A, Hajnal JV, Rueckert D. Multi-atlas based segmentation of brain images: atlas selection and its effect on accuracy. Neurolmage 2009;46:726-38.

[8] Ashburner J, Friston KJ. Unified segmentation. Neurolmage 2005;26:839-51.

[9] Fischl B, van der Kouwe A, Destrieux C, Halgren E, Segonne F, Salat DH, et al. Automatically parcellating the human cerebral cortex. Cerebral cortex (New York, NY : 1991) 2004;14:11-22.

[10] Heckemann RA, Keihaninejad S, Aljabar P, Rueckert D, Hajnal JV, Hammers A, et al. Improving intersubject image registration using tissue-class information benefits robustness and accuracy of multiatlas based anatomical segmentation. Neurolmage 2010;51:221-7.

[11] Babalola KO, Cootes TF, Twining CJ, Petrovic V, Taylor CJ. 3D brain segmentation using active appearance models and local regressors. Medical image computing and computer-assisted intervention : MICCAI - International Conference on Medical Image Computing and Computer-Assisted Intervention 2008;11:401-8.

[12] Kelemen A, Szekely G, Gerig G. Elastic modelbased segmentation of 3-D neuroradiological data 
sets. leee Transactions on Medical Imaging 1999;18:828-39.

[13] Leventon ME, Faugeras O, Grimson WEL, Wells WM. Level set based segmentation with intensity and curvature priors2000.

[14] Yushkevich PA, Piven J, Hazlett HC, Smith RG, Ho S, Gee JC, et al. User-guided 3D active contour segmentation of anatomical structures: Significantly improved efficiency and reliability. Neuroimage 2006;31:1116-28.

[15] Yazdani S, Yusof R, Karimian A, Pashna M, Hematian A. Image segmentation methods and applications in MRI brain images. IETE Technical Review 2015;32:413-27.

[16] Marroquin JL, Vemuri BC, Botello S, Calderon E, Fernandez-Bouzas A. An accurate and efficient Bayesian method for automatic segmentation of brain MRI. IEEE transactions on medical imaging 2002;21:934-45.

[17] Everitt BS, Landau S, Leese M, Stahl D. Miscellaneous clustering methods. Cluster Analysis, 5th Edition 2011:215-55.

[18] Karimian A, Yazdani S, Askari M. Reducing the absorbed dose in analogue radiography of infant chest images by improving the image quality, using image processing techniques. Radiation protection dosimetry 2011;147:176-9.

[19] Kang D, Shin SY, Sung CO, Kim JY, Pack J-K, Choi HD. An improved method of breast MRI segmentation with Simplified K-means clustered images. Proceedings of the 2011 ACM Symposium on Research in Applied Computation: ACM; 2011. p. 22631.

[20] Balafar M. Fuzzy C-mean based brain MRI segmentation algorithms. Artificial Intelligence Review 2014:1-9.

[21] Gao Y, Mas JF, Kerle N, Navarrete Pacheco JA. Optimal region growing segmentation and its effect on classification accuracy. International journal of remote sensing 2011;32:3747-63.

[22] Asl AA, Soltanian-Zadeh H. Constrained optimization of nonparametric entropy-based segmentation of brain structures. Biomedical Imaging: From Nano to Macro, 2008 ISBI 2008 5th IEEE International Symposium on: IEEE; 2008. p. 41-4.

[23] Tsai A, Wells W, Tempany C, Grimson E, Willsky A. Mutual information in coupled multi-shape model for medical image segmentation. Medical Image Analysis 2004;8:429-45.

[24] Vineetha G, Darshan G. Level set method for image segmentation: a survey. IOSR J Comput Eng 2013;8:74-8.
[25] Rohlfing T, Zahr NM, Sullivan EV, Pfefferbaum A. The SRI24 multichannel atlas of normal adult human brain structure. Human brain mapping 2010;31:798819.

[26] Igual L, Soliva JC, Hernández-Vela A, Escalera S, Jiménez $X$, Vilarroya $O$, et al. A fully-automatic caudate nucleus segmentation of brain MRI: application in volumetric analysis of pediatric attentiondeficit/hyperactivity disorder. Biomedical engineering online 2011;10:105.

[27] Kalinić $\mathrm{H}$. Atlas-based image segmentation: A Survey. 2009.

[28] Babalola KO, Patenaude B, Aljabar P, Schnabel J, Kennedy D, Crum W, et al. An evaluation of four automatic methods of segmenting the subcortical structures in the brain. Neurolmage 2009;47:1435-47.

[29] Babalola KO, Patenaude B, Aljabar P, Schnabel J, Kennedy D, Crum W, et al. Comparison and evaluation of segmentation techniques for subcortical structures in brain MRI. International Conference on Medical Image Computing and Computer-Assisted Intervention: Springer; 2008. p. 409-16. 